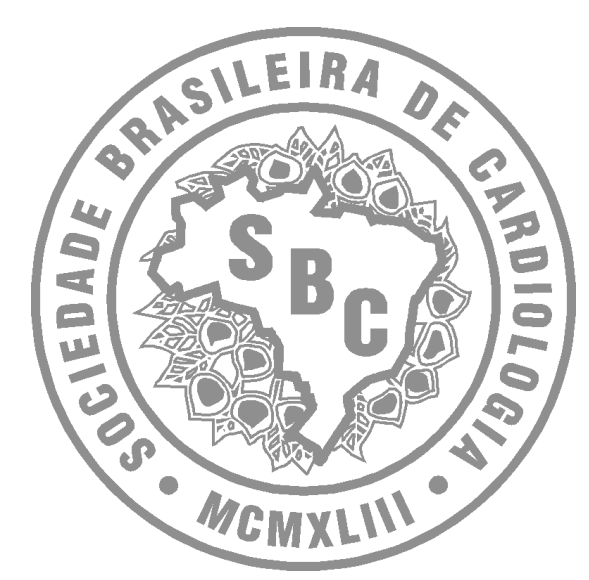

\title{
DIRETRIZES PARA HABILITAÇÃO DE CENTROS DE TREINAMENTO E PARA OBTENÇÃO DE CERTIFICAÇÃO EM HEMODINÂMICA E CARDIOLOGIA INTERVENCIONISTA
}

\author{
Sociedade Brasileira de Hemodinâmica e Cardiologia Intervencionista \\ Sociedade Brasileira de Cardiologia
}

\author{
Editores \\ Eulógio Emílio Martinez Filho \\ Luiz Alberto Piva Mattos \\ Paulo R. A. Caramori \\ Editores Associados \\ Adriano Mendes Caixeta \\ Ari Mandil \\ Cláudia M. Rodrigues Alves \\ Cesar A. Esteves \\ Fábio Sândoli de Brito Júnior \\ Fausto Feres \\ J osé Klauber Roger Carneiro \\ Newton Fernando Stlader de Souza Filho
}

Coordenador de Normatizações e Diretrizes da SBC 
Diretrizes para Habilitação de Centros de Treinamento e para Obtenção de Certificação em Hemodinâmica e Cardiologia Intervencionista

\section{Introdução}

Em 1977 foi criado o Departamento de Hemodinâmica e Angiocardiografia da Sociedade Brasileira de Cardiologia (SBC). Posteriormente foi fundada, em 1993, a Sociedade Brasileira de Hemodinâmica e Cardiologia Intervencionista (SBHCl).

Ao longo de mais de 25 anos consecutivos de trabalho associativo e científico, a SBHCl acompanhou o crescimento exponencial e a aceitação, por parte da comunidade e dos médicos, da realização de exames diagnósticos hemodinâmicos e angiográficos, elucidando as diversas formas de doenças cardiovasculares, congênitas ou adquiridas. Também participou da introdução e estabelecimento de diversas técnicas de diagnóstico e tratamento de múltiplas formas de apresentação das doenças cardiovasculares no Brasil.

A SBHCl sempre manifestou seu interesse na vigilância constante relacionada à certificação de novos profissionais, aptos à execução destes procedimentos em nosso país. 0 quadro associativo exibe crescimento contínuo e ininterrupto, sendo estimado que, até o final de 2005, estejam integrados à $\mathrm{SBHCl}$ aproximadamente 1.000 médicos cardiologistas intervencionistas.

A evolução observada na prática da cardiologia intervencionista determinou a necessidade da ampliação dos conhecimentos teóricos e práticos para a formação dos profissionais da área. A Associação Médica Brasileira (AMB) o Conselho Federal de Medicina (CFM) e a Comissão Nacional de Residência Médica (CNRM) reconheceram a diferenciação de treinamento necessária para a prática da especialidade, estabelecendo o certificado de área de atuação em hemodinâmica e cardiologia intervencionista, a partir do ano de 2001, definindo-a como uma área de atuação da cardiologia.

Contudo, as regras determinadas pela AMB/CFM/CNRM para certificação em hemodinâmica e cardiologia intervencionista, bem como as estabelecidas nos estatutos da $\mathrm{SBHCl}$ para formação de um membro titular, não contemplam os avanços e a complexidade crescente, relacionados à prática atual, justificando a necessidade de atualização.

Este documento é o resultado de um consenso de seus editores. As diretrizes elaboradas têm por finalidade detalhar e atualizar critérios e fixar novas regras para a habilitação de Centros de Treinamento nesta área de atuação, assim como fixar critérios para formação de novos profissionais e para a obtenção do certificado de área de atuação em hemodinâmica e cardiologia intervencionista. As mesmas devem ser implementadas a partir das modificações que sejam necessárias nos estatutos da $\mathrm{SBHCl}$.

\section{Recomendações para Habilitação dos Centros de Treinamento em Hemodinâmica e Cardiologia Intervencionista}

Até o final de 2002, 41 centros estavam habilitados pela $\mathrm{SBHCl}$ para o treinamento em hemodinâmica e cardiologia intervencionista. Os centros existentes e os novos interessados deverão seguir os critérios expostos a seguir, com a finalidade de requisitarem ou manterem o seu credenciamento como Centros de Forma-

\section{Recomendações para Habilitação de Centros de Treinamento}

1. Pertencer ou ser afiliado a um programa de residência médica em cardiologia, credenciado pela Comissão Nacional de Residência Médica, conforme resolução do Conselho Federal M edicina (Resolução 1666/2003/CFM).

2. Possuir ou ser associado a serviço de cirurgia cardíaca e vascular.

3. Enviar para a Central Nacional de Intervenções Cardiovasculares (CENIC) o registro de todos os casos de intervenção cardiovascular percutânea realizados anualmente.

4. Realizar cateterismos cardiovasculares diagnósticos, sendo pelo menos 1.000 cateterismos cardíacos anuais, comprovados por declaração assinada do chefe do serviço, referentes aos dois últimos anos de atividade.

5. Realizar intervenções cardiovasculares percutâneas, incluindo intervenções coronarianas, vasculares, congênitas e valvulares, sendo, pelo menos, 400 intervenções coronarianas percutâneas, comprovadas por meio de relatório da CENIC, referentes aos dois últimos anos.

6. Possuir, no mínimo, dois (02) preceptores, com certificado de área de atuação em Hemodinâmica e Cardiologia Intervencionista, membros titulares da $\mathrm{SBHCl}$ há pelo menos cinco (05) anos, em dia com suas obrigações associativas. Cada preceptor deverá comprovar a manutenção de proficiência, através dos registros da CENIC, demonstrando um mínimo de 75 intervenções cardiovasculares por ano, nos últimos 2 anos.

7. É recomendação desta Diretriz, que o número de vagas disponibilizadas por ano em um centro de formação esteja vinculado ao número de procedimentos anuais por centro e ao número de preceptores credenciados: para cada aluno, um preceptor e, no mínimo, 250 procedimentos intervencionistas percutâneos realizados no ano anterior.

8. Coordenador do Programa deverá ser um dos preceptores, que será o responsável, junto à $\mathrm{SBHCl}$, pela observância destas recomendações

9. A SBHCl será a responsável pela avaliação dos centros e verificação do cumprimento destas determinações, tanto na renovação como no credenciamento de novos centros de treinamento.

10. Os centros já habilitados terão o prazo de 18 meses, a partir da publicação destas recomendações, para solicitarem seu recadastramento como Centros de Treinamento, a fim de manterem a sua habilitação.

11. Serão realizadas verificações periódicas, visando conferir o cumprimento destas recomendações. Os centros que as descumprirem estarão sujeitos à perda da sua habilitação. 0 comunicado será feito por escrito ao coordenador do programa. Nesses casos, os centros terão 90 dias para solicitarem uma nova avaliação, após a devida correção. Mediante a evidência de ajustes apenas parciais das anormalidades observadas, um novo período de 45 dias será efetivado. A persistência das incorreções promove a perda da habilitação do centro.

\section{Recomendaçõese Obrigações do Centro de Treinamento}

0 período de tempo determinado para o treinamento é de 24 meses consecutivos. Devem ser reservados 30 dias anuais para 
férias, aprimoramento científico, ou participação em congressos e encontros da especialidade. Situações inusitadas, que requerem o afastamento prolongado do aluno (como doença ou gravidez), deverão ser contornadas com o cumprimento de tempo adicional, no próprio centro, até a completa efetivação do treinamento. Não serão aceitas interrupções superiores a 12 meses, e o prazo máximo para a duração do treinamento deverá ser de até 36 meses.

O programa de treinamento deverá propiciar ao aluno formação completa com domínio de técnicas e conhecimentos relativos à intervenção cardiovascular. 01 a ano do programa deverá oferecer treinamento teórico-prático, fundamentalmente em noções básicas e procedimentos percutâneos diagnósticos. 020 ano deverá ser direcionado para o treinamento em procedimentos percutâneos terapêuticos. A formação exclusiva em cardiologia intervencionista para o diagnóstico e tratamento das cardiopatias congênitas será permitida àqueles médicos que possuam título de especialista em cardiologia da SBC e certificado de área de atuação em cardiologia pediátrica.

O programa de atividade teórica e prática, do qual o Coordenador deverá enviar cópia para a sede da $\mathrm{SBHCl}$, deverá incluir:

1. Participação direta, sob supervisão do preceptor, em cateterismos cardíacos e vasculares diagnósticos e em intervenções coronarianas e vasculares percutâneas. Durante o período de treinamento, recomenda-se que o aluno atue como primeiro operador sob supervisão em: a) 400 cateterismos cardíacos; b) 120 intervenções coronarianas percutâneas. Também é recomendada a participação ativa em angiografias diagnósticas e intervenções cardiovasculares percutâneas (vasculares, cardíacas congênitas e valvulares).

2. Participação periódica em reuniões clínicas e científicas sobre cardiologia clínica e intervencionista cardiovascular, que propiciem a adequada formação do aluno, compreendendo o curriculum teórico básico descrito no Anexo 1 .

\section{Pré-requisitos para Admissão de Alunos no Centro de Treinamento}

1. Inscrição no respectivo CRM.

2. Conclusão de dois (02) anos de residência médica em cardiologia, credenciada pela Comissão Nacional de Residência Médica ou pela SBC, ou título de especialista em cardiologia pela $\mathrm{AMB} / \mathrm{SBC}$.

\section{Recomendações para Certificação em Hemodinâmica e Cardiologia Intervencionista e ao Comitê de Provas e Certificação da SBHCI}

Os candidatos à obtenção de Certificação em Hemodinâmica e Cardiologia Intervencionista deverão preencher os seguintes requisitos: 1. ser médico inscrito em CRM; 2. possuir título de especialista em cardiologia pela AMB/ SBC; 3 . conclusão do período de formação em Centro de Treinamento habilitado pela $\mathrm{SBHCl} ; 4$. aprovação nas provas teórica e prática realizadas pela $\mathrm{SBHCl} ; \mathbf{5}$. entrega dos devidos documentos e certificados na sede da $\mathrm{SBHCl}$.

Os candidatos à habilitação que efetivaram o seu treinamento no exterior deverão cumprir todos os critérios acima descritos, excetuando-se o item número 3. A comprovação da realização do

\section{ANEXO 1 Hemodinâmica e Cardiologia Intervencionista}

O programa de treinamento em Cardiologia Intervencionista deverá incluir:

1. Conhecimentos relativos a: a) fisiologia cardiovascular; b) mecanismos patológicos das doenças cardiovasculares, incluindo determinantes de aterosclerose e trombose; c) manifestações sistêmicas da aterosclerose e fatores de risco que contribuem para o seu desenvolvimento; d) diretrizes estabelecidas para modificação destes fatores de risco; e) fisiopatologia, manifestações clínicas, história natural, avaliação e manejo das doenças cardiovasculares; f) estados protrombóticos, incluindo distúrbios hereditários e adquiridos.

2. Noções básicas de radiação ionizante, formação da imagem, proteção radiológica.

3. Acessos vasculares - anatomia vascular; escolha e técnica de acesso arterial e venoso em múltiplos sítios (radial, braquial, femoral), técnicas para obtenção de hemostase e reconhecimento e tratamento de complicações de acesso vascular.

4. Registros pressóricos e medida de débito cardíaco - avaliação crítica da qualidade dos registros e seu funcionamento; reconhecimento das curvas pressóricas nas diferentes cavidades na fisiologia normal e em situações patológicas.

5. Contrastes iodados - tipos, dose, complicações e prevenção e tratamento de reações alérgicas e nefrotoxicidade a contraste.

6. Conhecimentos da anatomia radiológica cardíaca, coronariana e vascular e as devidas projeções angiográficas para realização de adequada dos procedimentos cardiovasculares.

7. Interpretação de imagens e angiografia quantitativa.

8. Medida do fluxo na circulação sistêmica e pulmonar; cálculo de shunts e resistências; cálculo de área valvar; monitorização das alterações hemodinâmicas em resposta a drogas

9. Fisiologia e fisiopatologia do fluxo coronariano - métodos de avaliação e interpretação de resultados (doppler-flow e pressure-wire).

10. Ultra-som intravascular - técnica de execução e interpretação de imagens.

11. Conhecimento técnico dos materiais utilizados para procedimentos diagnósticos e intervencionistas cardiovasculares.

12. Reconhecimento e manejo de complicações de cateterismo cardíaco e angiografia cardiovascular diagnóstica terapêutica.

13. Farmacologia aplicada a procedimentos percutâneos cardiovasculares medicações utilizadas no atendimento das urgências (parada cardiorrespiratória e anafilaxia), manejo de choque cardiogênico, de espasmo vascular, uso de antiplaquetários, anticoagulantes, trombolíticos, anti-anginosos e anti-hipertensivos.

14. Indicações e contra-indicações de cateterismo cardíaco e angiografia cardiovascular diagnóstica.

15. Diagnóstico angiográfico das principais doenças cardiovasculares congênitas na criança e no adulto.

16. Indicações, contra-indicações, técnica e limitações dos diversos procedimentos terapêuticos em Cardiologia Intervencionista, incluindo: a) intervenção coronária e vascular; b) utilização de dispositivos de proteção distal; c) valvoplastias; d) alcoolização e embolização de artérias coronárias; e) retirada de corpos estranhos intravasculares;

17. Indicações, contra-indicações, técnica e limitações dos diversos procedimentos terapêuticos em Cardiologia Intervencionista congênita incluindo: a) septostomia atrial (as diversas técnicas) e valvoplastias; b) angioplastia das artérias pulmonares e outras artérias e veias; c) aortoplastia e stent aórtico; d) radiofreqüência para perfuração valvar; e) técnicas de oclusão: embolizações, dispositivos de oclusão de defeitos cardíacos.

18. Aspectos técnicos peculiares de cada dispositivo de intervenção percutânea cardiovascular.

19. Análise crítica dos estudos publicados (medicina baseada em evidências).

número de procedimentos será verificada mediante documento assinado pelo Chefe de Serviço do respectivo local de treinamento. A qualificação e a idoneidade do Centro de Formação não brasileiro é de jurisprudência da $\mathrm{SBHCl}$. Não caberá recurso diante do seu resultado. A prova prática deverá ser realizada em um Centro de Treinamento brasileiro, sendo a escolha facultada ao candidato.

I. Prova teórica: A prova teórica é realizada anualmente, conforme edital publicado pela SBHCl. É preparada pela Comissão Científica da $\mathrm{SBHCl}$ ou por comissão específica por ela delegada, 
sendo composta de perguntas com respostas objetivas e discussão de casos clínicos. A prova deverá avaliar o domínio de conhecimentos relativos a todos os tipos de intervenção cardiovascular, incluindo cardiopatias congênitas e adquiridas e doença coronariana e vascular. Uma prova teórica relacionada ao diagnóstico e ao tratamento percutâneo das cardiopatias congênitas será oferecida àqueles que realizaram treinamento dedicado à área. A nota necessária para aprovação é maior ou igual a sete $(7,0)$. A avaliação de curriculum vitae também poderá ser realizada, conforme determinado pelo edital divulgado pela $\mathrm{SBHCl}$.

As exigências requeridas aos candidatos à realização da prova teórica são as seguintes: 1 . inscrição definitiva no Conselho Regional de Medicina; 2 . conclusão de dois (02) anos de residência médica em cardiologia clínica, credenciada pela Comissão Nacional de Residência Médica ou pela SBC, título de especialista em cardiologia pela $A M B / S B C ; 3$. conclusão do primeiro ano de treinamento em Intervenção Cardiovascular em Centro de Treinamento credenciado pela $\mathrm{SBHCl}$, conforme certificado do coordenador local; 4. certificado de área de atuação em cardiologia pediátrica, para os candidatos a prova teórica específica de cardiologia intervencionista congênita; 5. pagamento de taxa de inscrição estipulada pela $\mathrm{SBHCl}$.

II. Prova prática: As exigências requeridas aos candidatos para a realização da prova prática são as seguintes: 1 . inscrição no CRM ; 2. aprovação prévia na prova teórica da $\mathrm{SBHCl}$; 3. comprovação de conclusão do Programa de treinamento em Centro habilitado pela $\mathrm{SBHCl}$, conforme certificado emitido pelo coordenador do programa; 4. apresentação de certificado comprovando a participação direta como primeiro operador em 400 cateterismos cardíacos e angiografias diagnósticos; 5. comprovação de participação como primeiro operador em 120 intervenções coronarianas percutâneas; 6. pagamento da taxa de inscrição estipulada pela $\mathrm{SBHCl}$.

Ila. Realização e julgamento da prova prática: A solicitação da prova prática deverá ser efetuada pelo aluno, por escrito, à secretaria da $\mathrm{SBHCl}$. Após a solicitação e análise do cumprimento dos quesitos necessários, a SBHCl realizará a prova dentro de um período de até 60 dias.

0 aluno deve realizar, como primeiro operador independente, uma intervenção coronariana ou cardiovascular percutânea, de acordo com as seguintes normas: 1. A prova deverá ser realizada em um Centro de Treinamento credenciado pela SBHCl. O local e hora da prova serão divulgados pela $\mathrm{SBHCl}$. 2. A banca examinadora será composta de três (03) membros titulares da SBHCI há, pelo menos, cinco (05) anos, em dia com suas obrigações associativas, sendo designada pela $\mathrm{SBHCl}$. Em nenhuma hipótese, haverá recurso às decisões da banca examinadora. 3. Os membros da banca examinadora podem participar de até duas provas práticas, de alunos distintos, em um mesmo Centro de Treinamento, no mesmo dia. 4. Não será permitida a participação na banca examinadora de membros titulares pertencentes ao Centro de Treinamento do aluno. 5. Um dos preceptores que participou da formação do aluno deverá estar à disposição da banca examinadora para eventuais esclarecimentos. 6 . A banca examinadora registrará em ata a realização da prova, que será encaminhada, sob sigilo, à $\mathrm{SBHCl}$. 7. 0 resultado da prova é categórico, aprovado ou reprovado. 0 resultado será enviado por escrito, no prazo de até 30 dias após sua realização, por meio de comunicado oficial da SBHCl. 8. $\mathrm{Na}$ evidência de reprovação: a) a $\mathrm{SBHCl}$ informará ao aluno, por escrito, as razões da reprovação, auxiliando nas correções e aprimoramentos das deficiências apresentadas; b) o aluno poderá repetir a prova, sendo sugerido um treinamento adicional em Centro de Treinamento habilitado pela $\mathrm{SBHCl}$. 9. As eventuais expensas relacionadas ao comparecimento dos membros da banca examinadora no Centro de Treinamento serão de responsabilidade da $\mathrm{SBHCl}$ (passagens e hospedagem).

IIb. Recomendações para a banca examinadora da prova prática: 0 candidato, ao realizar uma intervenção percutânea coronariana ou cardiovascular de forma independente, como primeiro operador, deverá ser avaliado pela banca examinadora quanto a três quesitos: conhecimentos, habilidades e atitudes.

1. Conhecimentos: a) definição da anatomia cardiovascular; b) indicações de procedimentos diagnósticos e terapêuticos; c) manejo clínico no período pré e pós-procedimento; d) escolha da via de acesso, agentes de contraste e instrumental mais adequado para a realização do procedimento; e) complicações potenciais e o seu tratamento; f) estimativa de sucesso e preditores de insucesso a curto e longo prazo para o procedimento.

2. Habilidades: a) tempo de gasto de fluoroscopia para a realização do procedimento; b) rigor técnico; c) capacidade de contornar eventuais dificuldades; d) utilização adequada da farmacologia adjunta; e) conduta para finalização do procedimento e hemostasia da via de acesso; f) prescrição médica após o procedimento e recomendações para a alta hospitalar.

3. Atitudes: a) pontualidade; b) relação médico-paciente; c) relação com enfermagem e auxiliares de sala.

A banca examinadora registrará o resultado da prova em ata, indicando se o candidato foi aprovado ou reprovado. Em caso de reprovação, deverão ser indicados os quesitos em que o desempenho do candidato foi considerado insuficiente. Esta ata deverá, então, ser encaminhada, sob sigilo, à SBHCl.

\section{Bibliografia consultada}

1. Hirshfeld J W, Ellis SG, Faxon DP, et al. Recommendations for the assessment and maintenance of proficiency in coronary interventional procedures. Statement of the American College of Cardiology. J Am Coll Cardiol. 1998, 31: 722-43.

2. Ruiz CE, Mullins AP, Rochini WAK, et al. Core Curriculum for the training of pediatric invasive/interventional cardiologists: Report of the Society for cardiac angiography and interventions committee on pediatric cardiology training standards. Cath and Cardiov Diagn. 1996; 37: 409-24.

3. Hodgson J M cb, Tommaso CL, Watson RM, Weiner BH. Core curriculum for the training of adult invasive cardiologists: Report of the society for Cardiac Angiogra- phy and Interventions Committee on training standards. Cathet and Cardiov Diagn. 1996; 37: 392-408.

4. Beller GA, Bonow RO, Fuster V. Core Cardiology Training in Adult Cardiovascular Medicine (COCATS). American College of Cardiology- www. acc. org, 2002.

5. J acobs AK, Faxon DP, Hirshfeld J W, Holmes DR. Core cardiology training in adult cardiovascular medicine (COCATS) - Task force 3: Training in diagnostic cardiac catheterization and interventional cardiology. American College of Cardiology www. acc.org, 2002.

6. Smith SC J r, Dove J T, J acobs AK, et al. ACC/AHA guidelines for percutaneous coro- 
Diretrizes para Habilitação de Centros de Treinamento e para Obtenção de Certificação em Hemodinâmica e Cardiologia Intervencionista nay intervention: a report of the American College of Cardiology/American Heart Association Task Force on Practice Guidelines (Committee to revise the 1993 guidelines for percutaneous transluminal coronary angioplasty). J Am Coll Cardiol. 2001; 37: 2239i-Ixvi.

7. Hirshfeld J W J r, Banas J S, Cowley M, et al. American College of Cardiology Training Statement on Recommendations for the Structure of an Optimal Adult Interventional Cardiology training Program - A report of the American College of

Cardiology Task Force on Clinical Expert Consensus Documents. J Am Coll Cardiol. 1999; 34:2141-7.

8. Spittell J A J r, Creager MA, Dorros G, et al. Recommendations for Peripheral transluminal Angioplasty: Training and facilities. American College of Cardiology Peripheral Vascular disease committee. J Am Coll Cardiol 1993; 21: 546-8.

9. Eisemberg MJ, St Claire DA, Mak KH, Ellis SG. Importance of case mix during training in interventional cardiology. Am J Cardiol. 1996; 77: 1010-13. 